\title{
A NEW APPROACH TO ACUTE OTITIS IN INFANCY
}

\author{
By Prof. Henryk Lewenfisz, M.D. \\ Surgeon in Charge, Otorhinolaryngological Clinic, University of Lodz, Poland
}

For almost a century the question of ear inflammation in infants has been the field of investigation and the theme of papers by otologists, paediatricians and pathologists. The reason for this is the unusual frequency of suppurative changes found post mortem in the middle ear of infants who have died with symptoms of a disease of the digestive or respiratory system. Whereas the examination of ears in living children resulted in pathological findings in $20-40$ per cent. of cases, in as many as 80-100 per cent. of post mortem examinations suppurative changes in the middle ear were demonstrated. Gruber, Zaufel and others believed these changes to be the end-result of putrefaction of embryonic masses. According to Aschoff these changes were caused by the amniotic fluid reaching the tympanic cavity during intrauterine movements of breathing. He thinks that this condition is not a true infective otitis media, but a suppuration caused by extraneous matter. Gomperz's opinion is that in addition to the contamination of the tympanic cavity by amniotic fluid, there may be an additional factor of haemorrhagic changes taking place in the mucous membrane of the cavum tympani during difficult labour.

Today there is no doubt that the post mortem changes are indeed pathological (Wittmack and others). The disproportion between the results of clinical examination and the post mortem pathological findings is due to the absence of signs and the difficulty of diagnosis in cases of otitis in early infancy. The younger the child the greater the difficulties and the more general symptoms overshadow the local ones. Laboratory methods are of little help in diagnosing infantile otitis media, there is no characteristic blood picture in this condition and there is no typical temperature curve. The X-ray picture-in the first two years of life is also of very little diagnostic value. A careful observation of the sick child is much more helpful. The well known symptoms and signs :-restlessness, intermittent sucking while feeding, the rubbing of the ear with the hand or against the pillow, and tenderness over the tragus are not entirely dependable. This is especially so in the presence of generalized toxaemia and in the marasmic child.

Suppurative otitis media can be divided into two groups, the first follows upper respiratory tract infections and is relatively easy to diagnose. - The prognosis in these cases is good and the treatment conservative. The second group consists of the latent otitis, called by different authors otitis media concomitans, latens, occulta or pseudo-otitis. 'This type occurs concurrently with other diseases and most frequently accompanies gastrointestinal and pulmonary infections. The relationship between this latent otitis and the gastrointestinal disturbances, the so-called summer diarrhoea, is still undecided. Is the purulent otitis media, found at post mortem examination with other conditions, a secondary affection or is it a separate and primary entity?

There is no definite difference in the symptoms of true gastroenteritis and cases of diarrhoea and vomiting. According to certain French, American and Hungarian authors the intestinal disturbance results from the latent aural infection (Odeaual, Marriott, Gyorgyi). This is also the opinion of the Moscow University 'Sick Children's Clinic (Sokolow). The Warsaw Clinic also believes in the interdependance of intestinal and aural symptoms (Michalowicz, Brokman). The intestinal upset accompanying the ear infection was called by Michalowicz, colitoxaemia.

The death rate in some epidemics of summer diarrhoea was as high as $80-100$ per cent., and the post mortem examination showed in all cases bilateral suppurative changes in the tympanic cavity and in the mastoid. The ear condition is diagnosed clinically in only 20-40 per cent. of the enterotoxicoses, making it advisable to perform a myringotomy in all doubtful cases. This, however, does not always produce a purulent discharge and the physician, having excluded the possibility of otitis media, may still be confronted on the post mortem table with evidence of suppurative mastoiditis. There are some cases in which the pathologist cannot determine the correlation between the general condition and the middle ear disease. In addults a generalized infection follows lateral sinus thrombosis. In infants thrombosis rarely occurs. The micro-organisms easily enter the bloodstream through the porous structure of the mastoid process, which is richly supplied with blood vessels. This causes a bacteraemia, and the absorption of their toxins, a toxaemia. There- 
fore only a microscopical examination in these cases can show that the origin of the generalized infection lies in the sub-epithelial venous reticulum of the tympanic cavity and mastoid cells.

The child reacts differently from an adult to infection. Defence against micro-organisms is developed on quite different immuno-biological principles. Lowered resistance plays a more important rôle in children than in adults. In addition there are anatomical differences between adults and children which account for some of the diagnostic difficulties. In adults the diagnosis is based mainly on the changes seen in the drum, a method that is often useless in children, particularly during the first weeks of life.

The tympanic membrane in infants forms an angle of only $10^{\circ}-20^{\circ}$ with the external meatus, is normally dull, does not show the cone of light and the ossicles are invisible during the first few weeks of life. As the Eustachian tube remains wide open in middle ear infection the pus easily finds its way to the nasopharynx. The lack of increased pressure cause's hardly any bulging of the drum, which, however, can be seen bulging and pink when a healthy child cries. The external meatus is flattened antero-posteriorly so that its walls may be in apposition. It is narrow, covered with wax or vernix caseosa. All this makes the diagnosis difficult. Many otologists resort to a myringotomy, but this may neither clinch the diagnosis nor have a lasting therapeutic effect. It would be a mistake to say that the ear is healthy when after a myringotomy one sees no discharge, or when one sees no pus the next day, since the incision in the drum heals readily. When the general condition of the child does not improve a second myringotomy may be considered. A diagnostic puncture of the drum before myringotomy will only show if there is infection in the tympanic cavity. If the result is negative then the mastoid antrum should be punctured.

Examination of some hundreds of children, both healthy and with ear disease, aged one day onwards, allowed me to find some new signs and to work out new diagnostic methods. In children with an intact drum I have almost always been able to find a localized patch of intense red colour on the superior or postero-superior meatal wall. This was often accompanied by bulging. Both signs are particularly well defined in those with unilateral disease. They may also be seen in cases with perforated drums.

In children up to the age of three years, the bony external meatus is limited to the tympanic ring. The skin meatus lies directly on the bone of the mastoid process. Henle's ridge (crista Henle) does not exist in infants. In its place there is the lamina spongiosa or cribrosa (Poirier, Testut). The small openings in it lead to the mastoid antrum. The reddening and/or prolapse of the posterior wall of the external meatus are caused by the inflammatory process taking place in the antrum. This is most valuable in the diagnosis of middle ear disease in infants. The topography of this particular region permits a diagnostic puncture of the antrum to be performed via the meatus.

In cases of swelling and/or reddening of the posterior meatal wall the paracentesis yields purulent, sero-purulent or serous fluid. Systematically performed paracentesis of the drum and puncture of the antrum in infants permits the discovery of exudate in the tympanic cavity, in the antrum, or in both. If there is an open connection between the two cavities, then during and immediately after the paracentesis of the drum the swelling of the posterior meatal wall subsides and the reddening fades. If the swelling persists the cavities are separate, the divide being at the aditus. Under these circumstances mastoiditis is diagnosed and a distinction can be made between two possibilities :- first when there is exudate in both cavities and second when the exudate is limited to the antrum only. Often exudate is found in both, often it differs in quality ; one being serous, the other purulent. The character of the inflammatory fluid changes frequently during the progress of the illness. The organisms in the two cavities may be different. Sometimes the fluid from the tympanic cavity has been sterile and that from the antrum contained pathogenic microorganisms.

Our procedure in suspected cases of otitis in infants is as follows :- When the picture of the tympanic membrane is blurred (this is the rule in the first few months of life) and the posterior meatal wall is reddened and prolapsed we perform a paracentesis of the tympanic cavity, and if the result is positive, this is followed by a myringotomy. After this the swelling subsides at least temporarily. If the swelling persists the next day and the child's general condition does not show signs of improvement we puncture the antrum. If the myringotomy opening has closed, and that occurs fairly frequently in small infants, we repeat the drum paracentesis and if necessary the myringotomy, or even the mastoid antrum puncture. This procedure has to be repeated as long as the constitutional and local symptoms persist.

Antrum tapping, repeated once or twice, usually leads to the formation of an antro-meatal fistula. There is a form of mastoiditis called by Gele mastoiditis fistulosa, which is a self-terminating disease with abscesses in the mastoid process 
draining through a fistula to the meatus. All our cases of antritis are therefore transformed into a fistulous mastoiditis which leads to cure without an antrotomy having to be performed. An antrotomy in infants affected by a severe systemic illness is a serious undertaking especially as otitis in the very young is usually bilateral. We had to perform an antrotomy only in those cases where repeated paracentesis did not produce a cure.

The point of approach to the mastoid antrum lies on the posterior meatal wall in its upper part $2-3 \mathrm{~mm}$. lateral to the periphery of the drum. When the wall is swollen we choose the point of maximal bulging. Only very rarely in marasmic children was no swelling visible. A medium sized needle with a trocar, $6 \mathrm{~cm}$. long, with the point shortly bevelled, and a $\mathrm{I} \mathrm{cm}$. syringe is used. After puncturing the skin the thin bony lamina is pierced without any resistance. If the puncture is positive one can aspirate $0.1-0.15 \mathrm{ml}$. of exudate. The bacteriological examination of the fluid obtained from the tympanic cavity and from the antrum showed the presence of haemolytic streptococcus in 35 per cent. of cases, staphylococcus aureus 25 per cent., pneumococci 12 per cent., diphtheroids 8 per cent. ; 20 per cent. were sterile. The clinical progress of the case depended in no way on the strain of iolated microorganism. I introduced this method in 1940 at the Sick Children's Department of the City Hospital of Lwow.

My investigations were started because of the enormous death-rate in children during epidemics of summer and autumn diarrhoea. The deathrate was over 85 per cent. and had not been lowered in spite of such usual treatment as myringotomy, blood transfusion, water diet, feeding on human milk and on therapeutic baby foods. Antrotomies performed in a series of cases did not lower the death incidence appreciably.

The treatment by puncture and aspiration of the mastoid antrum was tried on 300 babies, six weeks-ten months old, with dramatic results. Within a few hours of the puncture the clinical picture changed entirely. Vomiting subsided, the number of motions diminished, other toxaemic symptoms disappeared, and the child started putting on weight. ' Frequently the general condition of the patient deteriorated again after one-three days. Then we repeated the antral punction daily, then every second or third day depending on the child's condition. In many cases an antro-meatal fistula formed. The death-rate fell to 30 per cent., then to 20 per cent.

It is worth mentioning that children brought to hospital were usually in very poor condition and a large number of deaths occurred during the 24 hours after admission. We had to perform an antrotomy in four cases as the condition did not improve after several paracenteses. In these cases we found advanced osteolytic changes. Of these four children one died the day after the operation with hyperthermia, another died a few days later and the other two survived. The operations were done under local analgesia.

In 95 per cent. of our patients we found inflammatory exudate already present in the ear on the first day after admission. The remaining 5 per cent. developed inflammatory changes later. Suppurative otitis was found in all severe cases immediately, or developed later when the general condition deteriorated.

It still remains to be decided what is the interrelationship between gastrointestinal disturbances and otitis. Personally I believe that otitis is not the primary condition. It is one of a number of factors and it plays a dominant rôle in the production of toxaemia, thus influencing the prognosis.

\section{Conclusions}

The most important sign in infantile otitis apart from the appearance of the drum is a localized reddening of the postero-superior wall of the meatus.

The reddening and bulging of the posterosuperior wall is a sign of infection with the presence of exudate in the mastoid antrum. The bulging in infants as opposed to the prolapse of the postero-superior meatal wall in adults is not an indication for an antrotomy.

These two signs, with diagnostic puncture of the tympanic cavity and/or the mastoid antrum lead to the correct diagnosis and allow of suitable treatment in every case.

Puncture of the mastoid antrum is both a diagnostic and a therapeutic measure.

The present series of cases does not contain any treated with sulphonamides or penicillin. 